\title{
Communication
}

\section{Genomic Analyses of Weissella cibaria W25, a Potential Bacteriocin-Producing Strain Isolated from Pasture in Campos das Vertentes, Minas Gerais, Brazil}

\author{
Camila Gonçalves Teixeira ${ }^{1,2} \mathbb{D}$, Rafaela da Silva Rodrigues ${ }^{1,3} \mathbb{D}$, Ricardo Seiti Yamatogi ${ }^{3}$, Anca Lucau-Danila ${ }^{2}$, \\ Djamel Drider $^{2}\left(\mathbb{D}\right.$, Luís Augusto Nero ${ }^{3, *(\mathbb{D})}$ and Antônio Fernandes de Carvalho ${ }^{1, *}$
}

check for updates

Citation: Teixeira, C.G.; Rodrigues, R.d.S.; Yamatogi, R.S.; Lucau-Danila, A.; Drider, D.; Nero, L.A.; de Carvalho, A.F. Genomic Analyses of Weissella cibaria W25, a Potential Bacteriocin-Producing Strain Isolated from Pasture in Campos das Vertentes, Minas Gerais, Brazil. Microorganisms 2022, 10, 314. https://doi.org/10.3390/ microorganisms10020314 Academic Editors: Maurizio Ciani and Franca Rossi

Received: 23 November 2021 Accepted: 17 January 2022 Published: 28 January 2022

Publisher's Note: MDPI stays neutral with regard to jurisdictional claims in published maps and institutional affiliations.

Copyright: (c) 2022 by the authors. Licensee MDPI, Basel, Switzerland. This article is an open access article distributed under the terms and conditions of the Creative Commons Attribution (CC BY) license (https:// creativecommons.org/licenses/by/ $4.0 /)$.
1 InovaLeite-Laboratório de Pesquisa em Leite e Derivados, Departamento de Tecnologia de Alimentos, Universidade Federal de Viçosa, Viçosa 36570 900, MG, Brazil; camila.g.goncalves.ufv@gmail.com (C.G.T.); rafaelasilva_mts@hotmail.com (R.d.S.R.)

2 Unité Mixte de Recherche (UMR) Transfrontalière BioEcoAgro1158, Univ. Lille, INRAE, Univ. Liège, UPJV, YNCREA, Univ. Artois, Univ. Littoral Côte D'Opale, ICV_Institut Charles Viollette, 59000 Lille, France; anca.lucau@univ-lille.fr (A.L.-D.); djamel.drider@univ-lille.fr (D.D.)

3 InsPOA—Laboratório de Inspeção de Produtos de Origem Animal, Departamento de Veterinária, Universidade Federal de Viçosa, Viçosa 36570 900, MG, Brazil; ryamatogi@ufv.br

* Correspondence: nero@ufv.br (L.A.N.); antoniofernandes@ufv.br (A.F.d.C.)

\begin{abstract}
Weissella is a genus containing Gram-positive, heterofermentative bacteria belonging to the lactic acid bacteria (LAB) group. These bacteria are endowed with promising technological and antimicrobial attributes. Weissella cibaria W25 was isolated from a dairy environment where raw milk cheeses are produced. Therefore, we sequenced and assembled the W25 draft genome sequence, which consists of 41 contigs totaling $\sim 2.4 \mathrm{Mbp}$, with a G $+\mathrm{C}$ content of $45.04 \%$. Then we carried out a comprehensive comparative genomic analysis with $W$. cibaria 110 , known to produce the weissellicin 110 bacteriocin, and four other non-bacteriocin-producing $W$. cibaria strains.
\end{abstract}

Keywords: Weissella; genome sequencing; bacteriocins genes

\section{Introduction}

The study of microbial diversity in dairy and non-dairy environments plays a pivotal role in understanding the presence of these microorganisms in such ecosystems and their impact on the final product, especially when we refer to traditional and artisanal products. Each environment has unique and specific characteristics that favor and allow the development of different bacterial species [1].

Handmade cheeses and raw milk are considered potential sources of new strains of LAB [2]. The way these cheeses are made can determine the fermentation to be conducted by bacteria present in the grazing, animal skin, utensils, surfaces and other places that may come into contact with the cheese during production [3]. The study of the bacterial community present in artisanal cheeses revealed the presence of species that had not yet been related to cheeses and a high diversity of lactic bacteria with differentiated technological characteristics [4]. In addition, non-dairy environments such as grass, different types of silage and even animal skin have also been important sources of novel strains that have adapted and, therefore, can provide interesting features to be explored [5].

The diversity of Weissella isolated from dairy and non-dairy environments is of great interest for the enrichment in knowledge of this microorganism in the final products. The genus Weissella is composed of bacteria classified as Gram-positive, catalase-negative, nonspore forming, coccoid morphology or short bacilli. They belong to the group of LAB, mainly due to its production of lactic acid from the fermentation of carbohydrates [6]. The main purpose of this study is to announce and analyze the sequencing and annotation of the Weissella cibaria W25 genome and carry out a comprehensive comparative genomic 
analysis with $W$. cibaria 110, known to produce the weissellicin 110 bacteriocin, and four other non-bacteriocin-producing $W$. cibaria strains.

\section{Materials and Methods}

\subsection{Bacterial Strain}

The strain W25 was previously isolated from pasture sampled from a dairy farm located in the Campos da Vertentes region, in the southeast of the Minas Gerais state, Brazil. This strain was named "isolate id 25" by Teixeira et al. [7], identified as Weissella cibaria after sequencing of the gene $16 \mathrm{~S}$ rRNA and characterized as possessing a technological potential due to its ability to coagulate milk and produce diacetyl and non-proteolytic. Of note, the strain presented antimicrobial activity against a panel of Gram-positive and Gram-negative foodborne pathogens [7].

\subsection{Genome Sequencing and Assembly}

The whole genome of $W$. cibaria W25 was sequenced with Nextera technology by a whole-genome shotgun strategy using the MiSeq v3 machine (Illumina, San Diego, CA, USA) by Neoprospecta (Florianópolis, SC, Brazil). The trimming was performed using the Trimmomatic v.0.36 [8] and the Phred value $>20$. The raw read files were trimmed of adapter sequences and low-quality bases. After trimming, sequence reads were checked for quality using the fastQC v.0.11.5 [9] and then used for de novo genome assembly. Genome assembly was conducted by using MIRA Assembler v.4.9.6 [10], mode "genome, accurate". The assembling quality was determined with QUAST v.5.0.2 [11], and ContEst16S was used to check contamination [12].

\subsection{Genome Annotation and Analysis}

Gene prediction and annotation were performed using the Rapid Prokaryotic Genome Annotation (PROKKA) v.1.14.5 [13], executed with default parameters and also performed by RAST automated web server [14]. To identify secondary metabolite biosynthetic gene clusters and bacteriocins, we used the antiSMASH v.6.0 [15] and the BAGEL4 webserver [16]. Moreover, the research for plasmid was evaluated by plasmidFinder [17] and the web tool PathogenFinder [18] was used to check the presence of potential virulence factors.

\subsection{Phylogenetic Analyses}

The identification of the genus and species was carried out using KmerFinder $[19,20]$ and the Type (Strains) Genome Server (TYGS) [21]. The phylogenetic trees were visualized and edited using the online tool iTol v.6 [22].

\subsection{Comparative Genomic}

To establish the relationship between $W$. cibaria W25 and other members of this species in the bacteriocin production context we selected four published non-bacteriocinsproducing $W$. cibaria strains and $W$. cibaria 110 known to produce the weissellicin 110 bacteriocin (Table 1). The genomes obtained from the GenBank were annotated using Prokka before being subjected to analyses in order to standardize the annotations.

The draft genome was submitted to the Type (Strains) Genome Server (TYGS) to confirm the genus and species. Moreover, to establish the genetic similarity between all strains, analysis was done with Digital DDH (DNA-DNA hybridization) similarities based on the GGDC (Genome-to-Genome Distance Calculator) web server, version 3.0 [23]. The core genome of each group was determined with OrthoVenn2 (e-value of $10^{-5}$ ) [24], and the CGView Server [25] was used for comparative genome analysis using BLAST with default parameters. 
Table 1. Comparison of the genomic feature of W. cibaria W25 with a bacteriocin-producing W. cibaria, strains 110, and four other non-bacteriocin-producing strains.

\begin{tabular}{|c|c|c|c|c|c|c|}
\hline Genome Feature & W25 & 110 & AB3b & ff3PR & $\mathrm{JCM} 12495^{\mathrm{T}}$ & MG1 \\
\hline Accession $^{a}$ & JAFNKE000000000 & LRRC00000000 & JWHT00000000 & JWHV00000000 & BJEF00000000 & JWHU00000000 \\
\hline Reference & This study & [26] & [27] & [27] & [28] & [27] \\
\hline Contigs & 41 & 18 & 88 & 60 & 25 & 44 \\
\hline Size $(\mathrm{pb})$ & $2,412,435$ & $2,347,049$ & $2,465,158$ & $2,357,128$ & $2,323,953$ & $2,436,232$ \\
\hline GC content $(\%)^{b}$ & 45.04 & 44.9 & 44.7 & 44.9 & 45.1 & 44.7 \\
\hline CDS & 2190 & 2209 & 2348 & 2228 & 2124 & 2284 \\
\hline tRNA & 84 & 76 & 67 & 70 & 77 & 62 \\
\hline rRNA & 15 & 5 & 7 & 5 & 9 & 4 \\
\hline tmRNA & 1 & 1 & 1 & 1 & 1 & 1 \\
\hline Repeat region & 1 & 1 & 0 & 0 & 0 & 0 \\
\hline
\end{tabular}

${ }^{\mathrm{a}}$ GenBank accession number; ${ }^{\mathrm{b}}$ using RAST program.

\subsection{Availability of Nucleotide Sequence Data}

This Whole Genome Shotgun project was deposited at DDBJ/ENA/GenBank under the accession JAFNKE000000000. The version described in this paper is version JAFNKE010000000. The raw sequencing data were submitted to the Sequence Read Archive (SRA) database under accession number SRR16076638.

\section{Results and Discussion}

\subsection{Genome Sequencing, Annotation and Analysis}

The genome features comparison between W. cibaria W25, W. cibaria 110, W. cibaria B3b, W. cibaria ffPR, W. cibaria JCM 12,495 and W. cibaria MG1 and the predicted genes are presented in Table 1 . The whole-genome sequencing of $W$. cibaria W25 resulted in maximum size of reads for the forward sequence of 305 and for the reverse sequence of 205 and with a total number of sequences of 2,906,916 bp. After genome assembly using the MIRA software, we obtained a draft genome with 41 contigs, $\mathrm{N}_{50} 202,649 \mathrm{bp}$ and maximum length of 331,445 bp (contigs over 500 bases).

The genome of $W$. cibaria W25 contains 2,412,435 bp, which is very similar to the genome of $W$. cibaria MG1, and slightly bigger than $W$. cibaria $110, W$. cibaria $\mathrm{ff} 3 \mathrm{PR}$ and W. cibaria JCM 12495. However, the GC contents are very similar between all of them, varying from 44.7 to $45.1 \%$. Genome annotation using Prokka identified a total of 2190 of coding DNA sequences (CDS) in the genome of $W$. cibaria W25. The amount of CDS were more abundant in our genome strains than in the genome of W. cibaria JCM 12,495 and less abundant than in the other genomes in this study. W. cibaria W25 presented the highest quantity of tRNA and rRNA with 11 copies of $5 S$ ribosomal RNA (rRNA) genes, 3 copies of $16 \mathrm{~S}$ and 1 single copy of 23S rRNA genes.

It is worth mentioning that draft genome assemblies often include an incorrect number of rRNA genes due to assembly artifacts.

No plasmid gene was detected by plasmidFinder in W. cibaria W25, and this input organism was predicted as a non-human pathogen by PathogenFinder. These results are an indication for the safe use of this strain for future human consumption as a probiotic or as a bioprotective culture in food, for example.

According to AntiSMASH, W. cibaria W25 possesses two putative bacteriocin gene clusters, one lassopeptide (MicJ25) and one RiPP-like bacteriocin_IIc. Of note, the Bagel4 software did not allow identification of any bacteriocin gene. Previously, we showed that $W$. cibaria W25 has a narrow spectrum of inhibition against the most common foodborne pathogens [7], reinforcing the idea that this strain is a suitable probiotic candidate. Li et al. (2017) [26] showed that W. cibaria 110 presents similar results showing a large spectrum of inhibition against other LAB, but the bacteriocin weissellicin 110, produced by W. cibaria 110, unlike most class II bacteriocins, has no inhibitory activity against Listeria monocytogenes. 


\subsection{Phylogenetic Analyses and Comparative Genomic}

Whole-genome-based phylogeny of the W. cibaria W25 was constructed using several genome sequences from Weissella species-type strains, including complete and draft genomes (Figure 1), by the TYGS web server. Figure 1 shows the formation of two major clusters, cluster one comprised six Weissella strains and cluster two all the other strains including the one in study. In addition, Figure 1 shows that W. cibaria W25 and W. cibaria JCM 12,495 are phylogenetically closely related. This result, along with the one from KmerFinder software, confirmed the genus Weissella and the species cibaria for the strain W25, as previously announced [7].

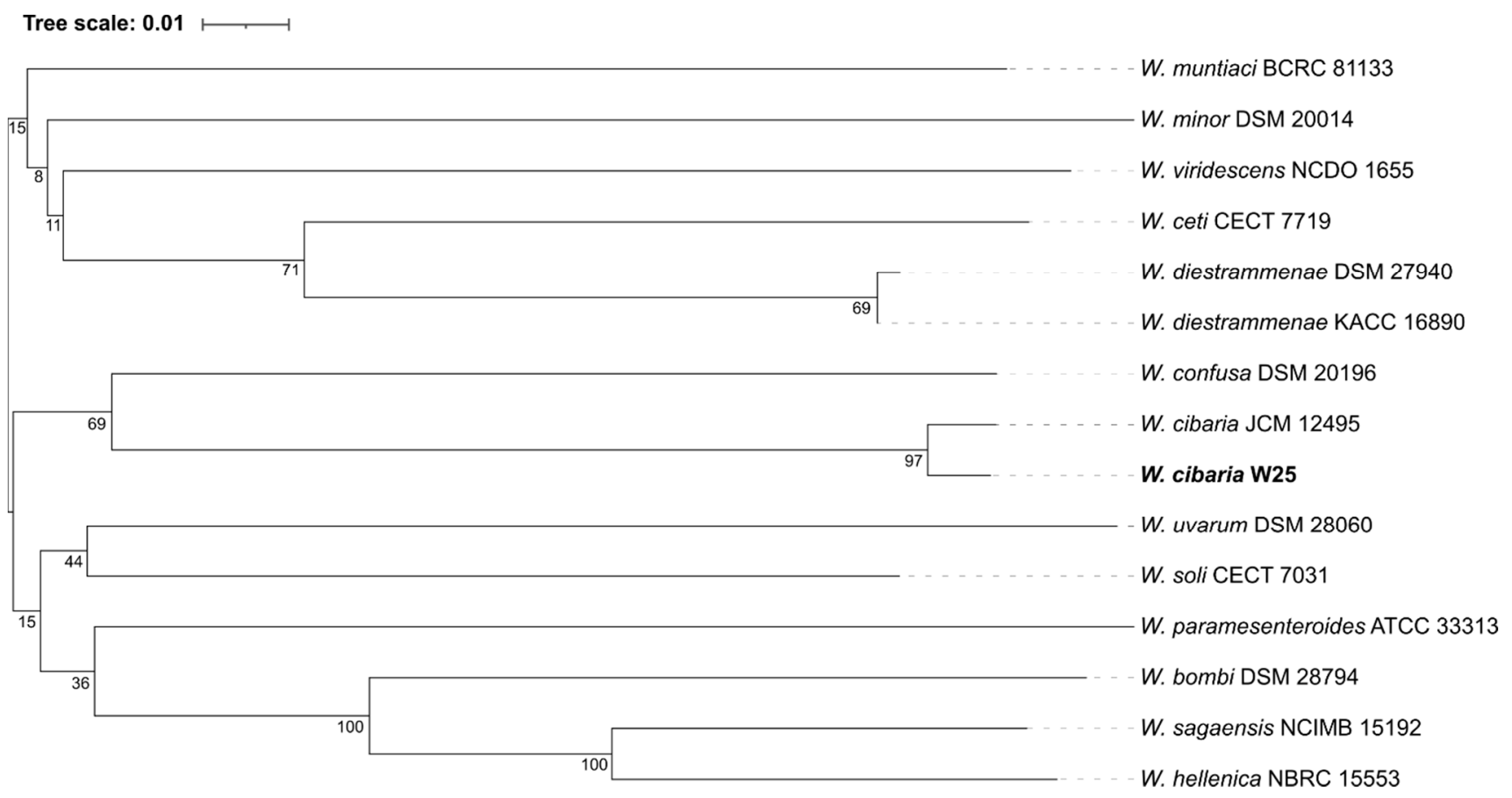

Figure 1. Tree inferred with FastME 2.1.6.1 [29] from GBDP distances calculated from genome sequences. The branch lengths are scaled in terms of GBDP distance formula $\mathrm{d}_{5}$. The numbers above branches are GBDP pseudo-bootstrap support values $>60 \%$ from 100 replications, with an average branch support of $55.9 \%$. The tree was rooted at the midpoint [30].

The digital DDH genomic similarity (Table 2) revealed that between the strains tested in this study W. cibaria W25 possesses more similarity with W. cibaria JCM 12,495 (87.10\%), a non-bacteriocin producer, and less similarity with $W$. cibaria $110(63.80 \%)$, a strain known as a bacteriocin producer.

Table 2. Genetic similarity between W. cibaria W25 and other strains using Digital DDH (DNADNA hybridization) similarities based on the GGDC (Genome-to-Genome Distance Calculator). The recommended formula 2 was chosen for the inferred distances.

\begin{tabular}{cccc}
\hline Strains & DDH & Prob. DDH $\geq \mathbf{7 0} \%$ & G + C Difference \\
\hline W. cibaria 110 & 63.8 & 63.95 & 0.18 \\
W. cibaria AB3b & 72.6 & 82.63 & 0.36 \\
W. cibaria ff3PR & 71.7 & 81.21 & 0.17 \\
W. cibaria JCM12495 & 87.1 & 94.69 & 0.11 \\
W. cibaria MG1 & 71.6 & 81.02 & 0.29 \\
\hline
\end{tabular}

According to the Venn diagram from OrthoVenn2, W. cibaria W25 shares the same quantity of protein cluster genes with $W$. cibaria 110 and W. cibaria JCM 12,495 (Figure 2) 
when comparing just the three of them. When we compared with all the strains used in this study, W. cibaria W25 shares more unique protein cluster genes [18] with W. cibaria 110 (Figure 3B) than with the others. Besides that, W. cibaria W25 shares 25 with all the nonbacteriocin-producing strains (Figure 3C). There were 1852 protein cluster genes conserved in all W. cibaria strains, and five of them are unique to W. cibaria W25 (Figure 3A) with 10 paralogs. Among the five protein clusters coding genes, the OrthoVenn 2 identified three of them, one related to the lipopolysaccharide biosynthetic process, one to the $\mathrm{O}$ antigen biosynthetic process and one to oxidoreductase activity. Li et al. (2017) [25] also compared W. cibaria 110 with four other strains (W. cibaria MG1, W. cibaria AB3b, W. cibaria ff3PR and $W$. cibaria KACC11862) and the comparative genomic analysis also showed the presence of unique genes that encoded the novel bacteriocin weissellicin 110 and defense system.
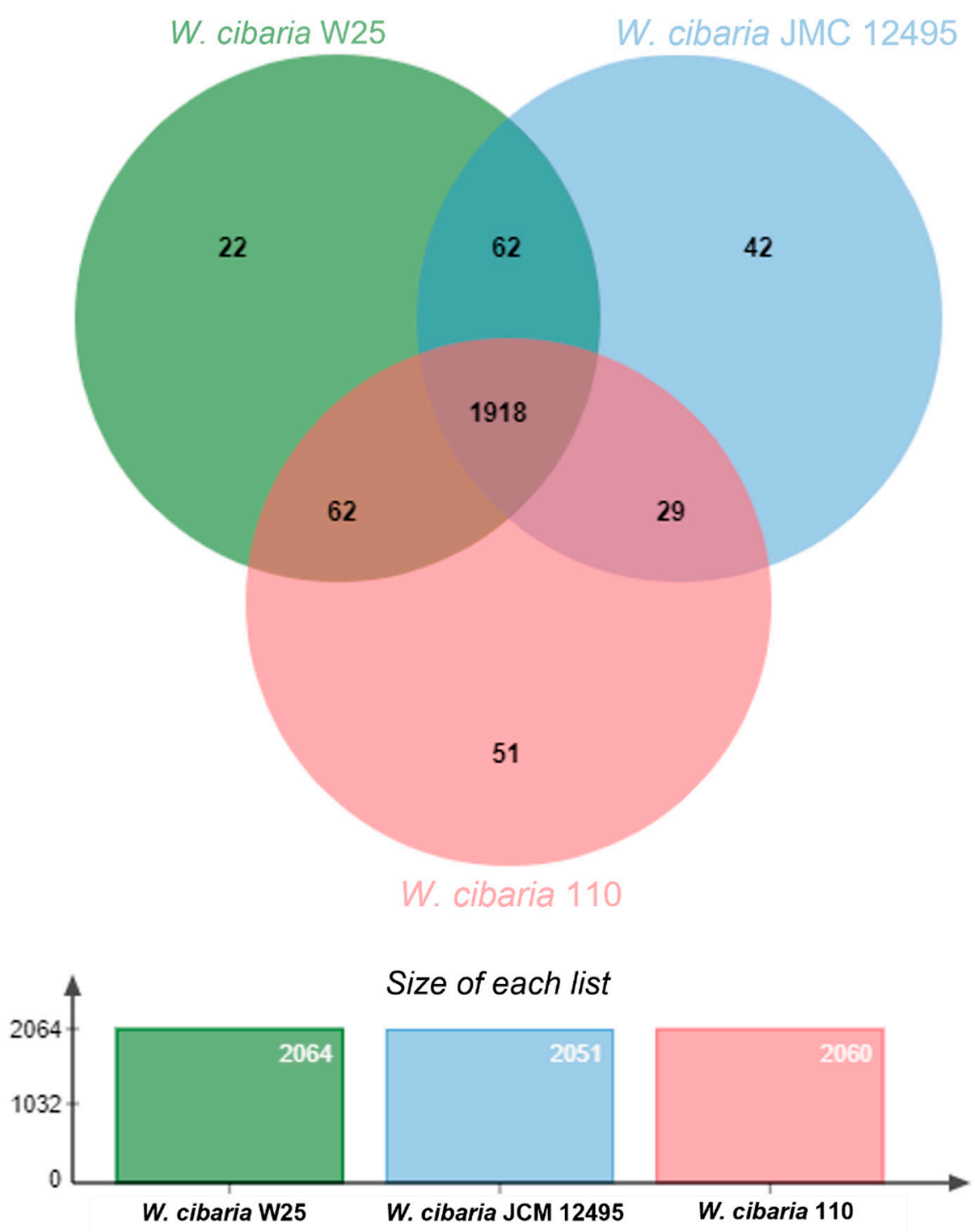

Number of elements: specific (1) or shared by 2, 3, ... lists

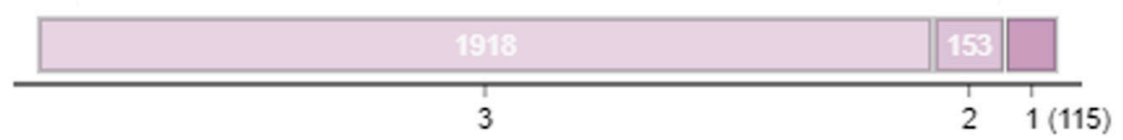

Figure 2. Venn diagram showing the protein coding genes or pseudogenes in each sequenced of $W$. cibaria W25, W. cibaria 110 and W. cibaria JMC 12495. Overlapped regions represent shared proteins and the numbers in the non-overlapped regions indicate the unique protein coding genes or pseudogenes. 
A
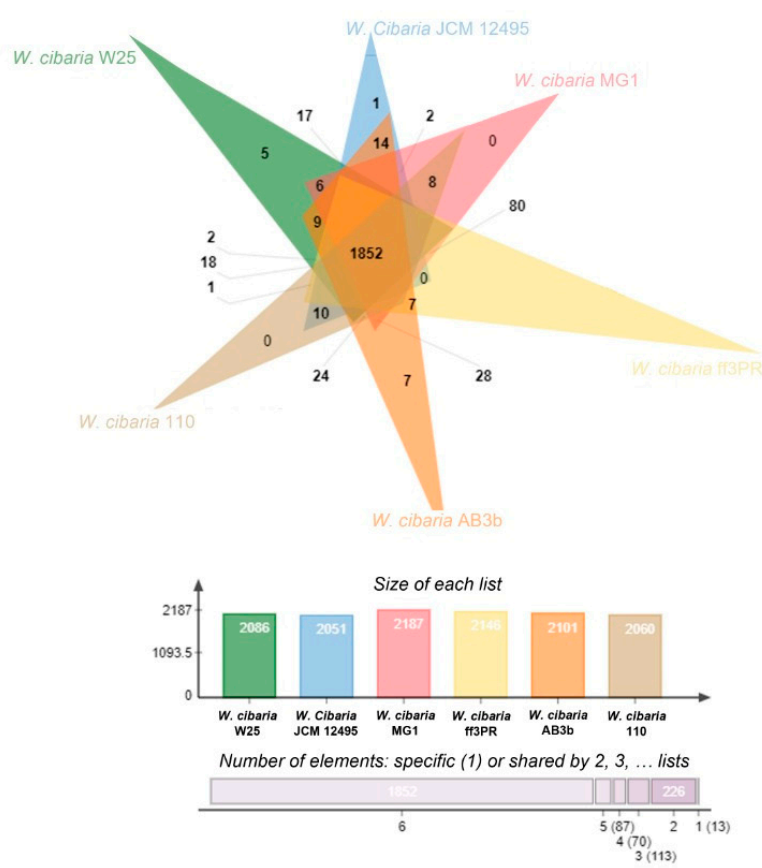

B

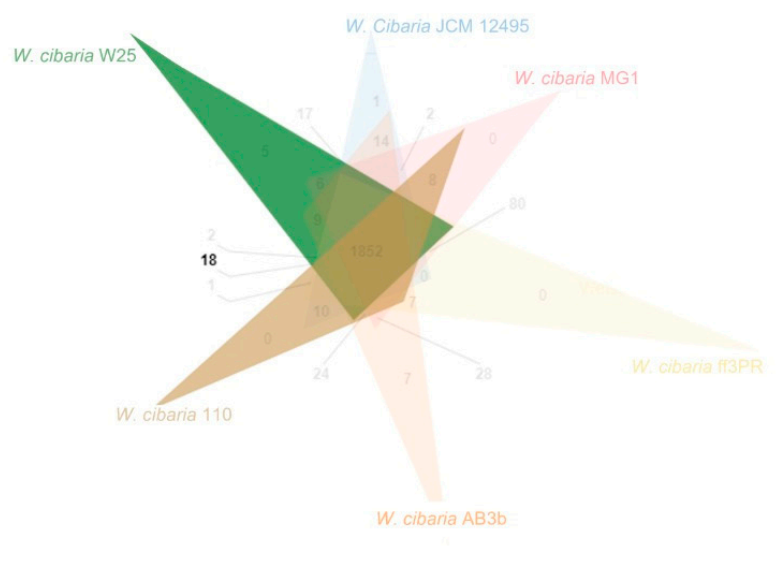

C

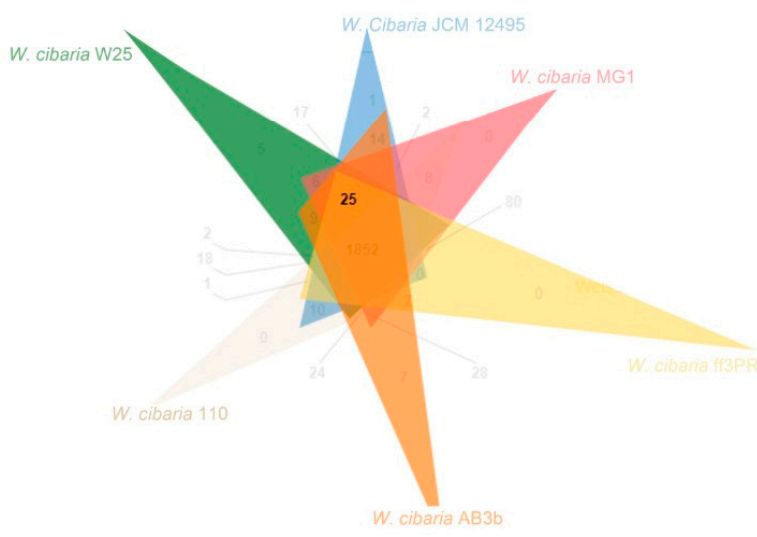

Figure 3. Venn diagrams showing the protein coding genes or pseudogenes of six W. cibaria strains. Overlapped regions represent shared proteins and the numbers in the non-overlapped regions indicate the unique protein coding genes or pseudogenes. (A) normal Venn diagram; (B) Venn diagram highlighting the cluster between $W$. cibaria W25 and W. cibaria 110; (C) Venn diagram highlighting the clusters between $W$. cibaria W25 and the non-bacteriocin-producing strains.

We constructed a comparative genetic map using the CGview server to help demonstrate the similarity between the strains (Figure 4). We used W. cibaria 110 as the reference for comparison because it is a well-known bacteriocin-producing strain. Moreover, gene confirmation for weissellicin 110 was performed by the BLASTn program at the National Center for Biotechnology Information (NCBI) using the deposited sequence under accession number LC010242 and the predicted genes of the genome from W. cibaria 110. We obtained results to query coverage and percent identity of $100 \%$. Among the regions that showed to be similar between $W$. cibaria W25 and $W$. cibaria 110, we observed that the strain under study presented a similar and more complete region when compared to the region containing weissellicin 110 than the non-bacteriocin-producing strains (Figure 4B). This indicates that among the shared genes between $W$. cibaria W25 and W. cibaria 110 there is one related to the production of bacteriocin but they are not completely the same, which indicates that perhaps in this region there are genes for a bacteriocin, however, they are distinct from weissellicin 110. 

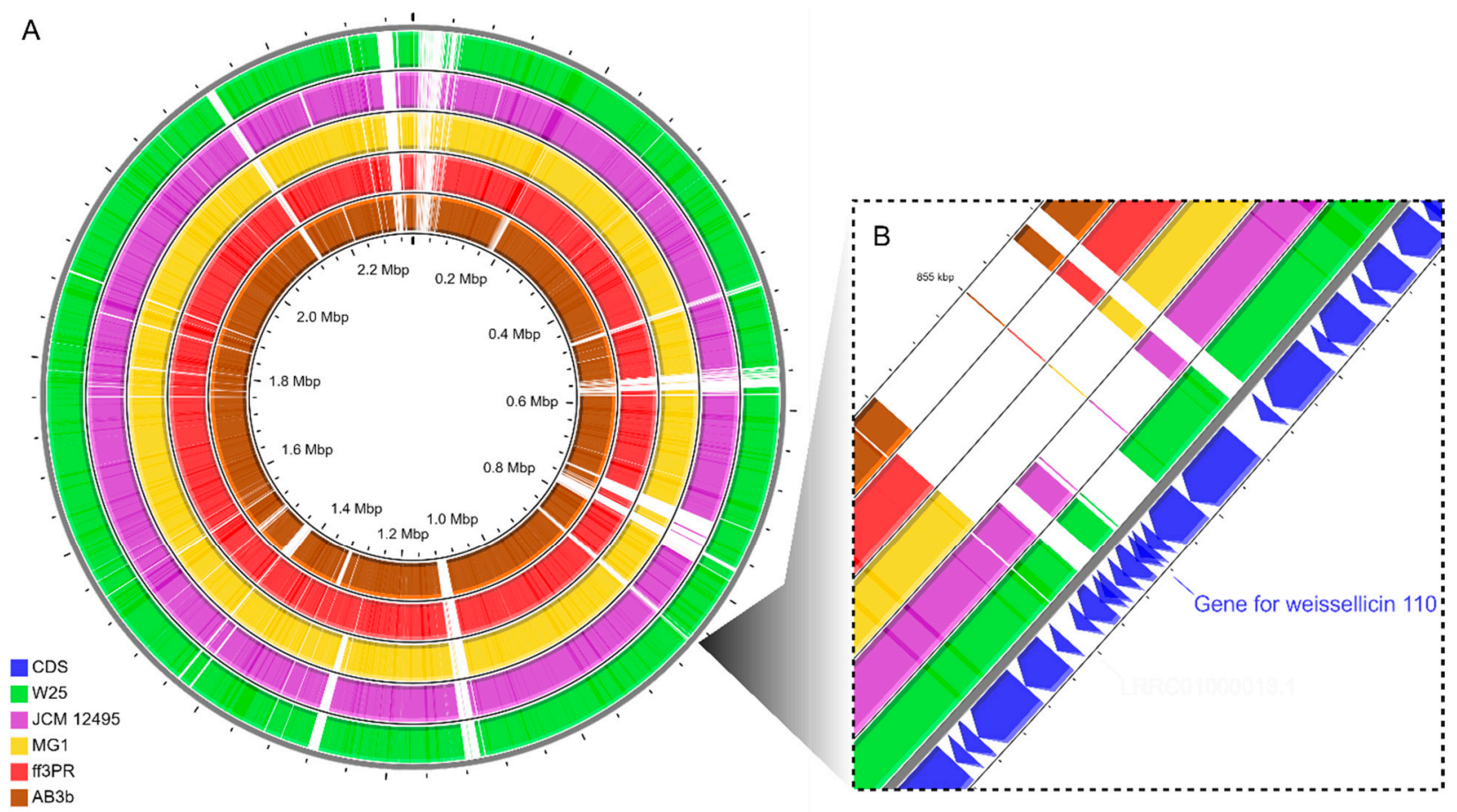

Figure 4. Comparative genome map generated using CGView server, showing a full circular map obtained using BLAST (A) and an expanded view of the weissellicin 110 region (B). The rings in (A) and (B) in green, purple, yellow, red and brown indicate the W. cibaria strains W25, JCM 12495, MG1, ff3PR and AB3b, respectively, that were compared with the $W$. cibaria 110 . The blue ring in (B) indicates the coding sequences (CDS) of the genome from W. cibaria 110.

\section{Conclusions}

According to the bioinformatics results obtained in this study, W. cibaria W25 has great potential to be used for human consumption since it was predicted as a non-human pathogen. In addition, despite W. cibaria W25 showing more genomic similarity with W. cibaria JCM 12,495 (according to DDH similarly), OrthoVenn2 showed that it has its unique protein cluster genes which can be related with the bacteriocin genes indicated by AntiSMASH, confirming the possibility of producing two different bacteriocins.

Author Contributions: C.G.T.: Conceptualization, Investigation, Methodology, Writing-original draft, Writing—review \& editing. R.d.S.R.: Conceptualization, Methodology, Writing—review \& editing. R.S.Y.: Data curation, Methodology, Validation, Writing-review \& editing. A.L.-D.: Methodology, Validation. D.D.: Conceptualization, Data curation, Formal analysis, Funding acquisition, Project administration, Resources, Supervision, Validation, Visualization, Writing-original draft, Writing -review \& editing. L.A.N.: Conceptualization, Data curation, Formal analysis, Funding acquisition, Project administration, Resources, Supervision, Validation, Visualization, Writing-original draft, Writing-review \& editing. A.F.d.C.: Conceptualization, Data curation, Formal analysis, Funding acquisition, Project administration, Resources, Supervision, Validation, Visualization, Writing —original draft, Writing -review \& editing. All authors have read and agreed to the published version of the manuscript.

Funding: This work was supported by Conselho Nacional de Desenvolvimento Científico e Tecnológico (CNPq, Brasília, DF, Brazil), Coordenação de Aperfeiçoamento de Pessoal de Nível Superior (CAPES, Brasília, DF, Brazil), and Fundação de Amparo à Pesquisa do Estado de Minas Gerais (FAPEMIG, Belo Horizonte, MG, Brazil). Research at Lille University is supported by CPER/FEDER Alibiotech grant 2016-2021.

Institutional Review Board Statement: Not applicable.

Informed Consent Statement: Not applicable. 


\section{Data Availability Statement: Not applicable.}

Conflicts of Interest: The authors declare no conflict of interest.

\section{References}

1. Finnegan, W.; Yan, M.; Holden, N.M.; Goggins, J. A review of environmental life cycle assessment studies examining cheese production. Int. J. Life Cycle Assess. 2018, 23, 1773-1787. [CrossRef]

2. e Silva, E.O.O.; Nespolo, C.R.; Sehn, C.P.; Pinheiro, F.C.; Stefani, L.M. Lactic acid bacteria with antimicrobial, proteolytic and lipolytic activities isolated from ovine dairy products. Food Sci. Technol. 2020, 40, 293-299. [CrossRef]

3. Pyz-Łukasik, R.; Knysz, P.; Gondek, M. Hygiene Quality and Consumer Safety of Traditional Short- and Long-Ripened Cheeses from Poland. J. Food Qual. 2018, 2018, 8732412. [CrossRef]

4. Tapia, J.A.A.; Escobar-Ramírez, C.M.; Tamplin, M.L.; Hernández-Iturriaga, M. Characterization of Bacterial Communities in Mexican Artisanal Raw Milk "Bola de Ocosingo" Cheese by High-Throughput Sequencing. Front. Microbiol. $2018,9,2598$. [CrossRef]

5. McAuliffe, O. Symposium review: Lactococcus lactis from nondairy sources: Their genetic and metabolic diversity and potential applications in cheese. J. Dairy Sci. 2018, 101, 3597-3610. [CrossRef]

6. Collins, M.; Samelis, J.; Metaxopoulos, J.; Wallbanks, S. Taxonomic studies on some leuconostoc-like organisms from fermented sausages: Description of a new genus Weissella for the Leuconostoc paramesenteroides group of species. J. Appl. Bacteriol. 1993, 75, 595-603. [CrossRef]

7. Teixeira, C.G.; Fusieger, A.; Martins, E.; de Freitas, R.; Vakarelova, M.; Nero, L.A.; de Carvalho, A.F. Biodiversity and technological features of Weissella isolates obtained from Brazilian artisanal cheese-producing regions. LWT 2021, 147, 111474. [CrossRef]

8. Bolger, A.M.; Lohse, M.; Usadel, B. Trimmomatic: A flexible trimmer for Illumina sequence data. Bioinformatics 2014, 30, 2114-2120. [CrossRef]

9. Andrews, S. FastQC: A Quality Control Tool for High Throughput Sequence Data. 2010. Available online: http://www. bioinformatics.babraham.ac.uk/projects/fastqc (accessed on 24 November 2021).

10. Chevreux, B.; Wetter, T.; Suhai, S. Genome Sequence Assembly Using Trace Signals and Additional Sequence Information. Ger. Conf. Bioinform. 1999, 99, 45-46.

11. Gurevich, A.; Saveliev, V.; Vyahhi, N.; Tesler, G. QUAST: Quality assessment tool for genome assemblies. Bioinformatics 2013, 29, 1072-1075. [CrossRef]

12. Lee, I.; Chalita, M.; Ha, S.-M.; Na, S.-I.; Yoon, S.-H.; Chun, J. ContEst16S: An algorithm that identifies contaminated prokaryotic genomes using 16S RNA gene sequences. Int. J. Syst. Evol. Microbiol. 2017, 67, 2053-2057. [CrossRef]

13. Seemann, T. Prokka: Rapid Prokaryotic Genome Annotation. Bioinformatics 2014, 30, 2068-2069. [CrossRef]

14. Aziz, R.K.; Bartels, D.; Best, A.A.; DeJongh, M.; Disz, T.; Edwards, R.A.; Formsma, K.; Gerdes, S.Y.; Glass, E.M.; Kubal, M.; et al. The RAST server: Rapid annotations using subsystems technology. BMC Genom. 2008, 9, 75. [CrossRef]

15. Blin, K.; Shaw, S.; Kloosterman, A.M.; Charlop-Powers, Z.; van Wezel, G.P.; Medema, M.H.; Weber, T. antiSMASH 6.0: Improving cluster detection and comparison capabilities. Nucleic Acids Res. 2021, 49, W29-W35. [CrossRef]

16. Van Heel, A.J.; De Jong, A.; Song, C.; Viel, J.; Kok, J.; Kuipers, O.P. BAGEL4: A user-friendly web server to thoroughly mine RiPPs and bacteriocins. Nucleic Acids Res. 2018, 46, W278-W281. [CrossRef]

17. Carattoli, A.; Zankari, E.; García-Fernández, A.; Voldby Larsen, M.V.; Lund, O.; Villa, L.; Møller Aarestrup, F.M.; Hasman, H. In Silico Detection and Typing of Plasmids using PlasmidFinder and Plasmid Multilocus Sequence Typing. Antimicrob. Agents Chemother. 2014, 58, 3895-3903. [CrossRef]

18. Cosentino, S.; Voldby Larsen, M.; Møller Aarestrup, F.; Lund, O. PathogenFinder-Distinguishing Friend from Foe Using Bacterial Whole Genome Sequence Data. PLoS ONE 2013, 8, e77302. [CrossRef]

19. Hasman, H.; Saputra, D.; Sicheritz-Ponten, T.; Lund, O.; Svendsen, C.A.; Frimodt-Møller, N.; Aarestrup, F.M. Rapid WholeGenome Sequencing for Detection and Characterization of Microorganisms Directly from Clinical Samples. J. Clin. Microbiol. 2014, 52, 139-146. [CrossRef]

20. Larsen, M.V.; Cosentino, S.; Lukjancenko, O.; Saputra, D.; Rasmussen, S.; Hasman, H.; Sicheritz-Pontén, T.; Aarestrup, F.M.; Ussery, D.W.; Lund, O. Benchmarking of Methods for Genomic Taxonomy. J. Clin. Microbiol. 2014, 52, 1529-1539. [CrossRef]

21. Meier-Kolthoff, J.P.; Göker, M. TYGS is an automated high-throughput platform for state-of-the-art genome-based taxonomy. Nat. Commun. 2019, 10, 1-10. [CrossRef]

22. Letunic, I.; Bork, P. Interactive tree of life (iTOL) v3: An online tool for the display and annotation of phylogenetic and other trees. Nucleic Acids Res. 2016, 44, W242-W245. [CrossRef]

23. Meier-Kolthoff, J.P.; Carbasse, J.S.; Peinado-Olarte, R.L.; Göker, M. TYGS and LPSN: A database tandem for fast and reliable genome-based classification and nomenclature of prokaryotes. Nucleic Acids Res. 2021, 50, D801-D807. [CrossRef]

24. Xu, L.; Dong, Z.; Fang, L.; Luo, Y.; Wei, Z.; Guo, H.; Zhang, G.; Gu, Y.Q.; Coleman-Derr, D.; Xia, Q.; et al. OrthoVenn2: A web server for whole-genome comparison and annotation of orthologous clusters across multiple species. Nucleic Acids Res. 2019, 47, W52-W58. [CrossRef]

25. Stothard, P.; Wishart, D.S. Circular genome visualization and exploration using CGView. Bioinformatics 2005, 21, 537-539. [CrossRef] 
26. Li, S.-W.; Chen, Y.-S.; Lee, Y.-S.; Yang, C.-H.; Srionnual, S.; Wu, H.-C.; Chang, C.-H. Comparative genomic analysis of bacteriocinproducing Weissella cibaria 110. Appl. Microbiol. Biotechnol. 2017, 101, 1227-1237. [CrossRef]

27. Lynch, K.; Arendt, E.K.; Lucid, A.; Lucey, B.; Sleator, R.; Coffey, A. Genomics of Weissella cibaria with an examination of its metabolic traits. Microbiology 2015, 161, 914-930. [CrossRef]

28. Bjorkroth, J.; Schillinger, U.; Geisen, R.; Weiss, N.; Hoste, B.; Holzapfel, W.H.; Korkeala, H.; Vandamme, P. Taxonomic study of Weissella confusa and description of Weissella cibaria sp. nov., detected in food and clinical samples. Int. J. Syst. Evol. Microbiol. 2002, 52, 141-148. [CrossRef]

29. Lefort, V.; Desper, R.; Gascuel, O. FastME 2.0: A Comprehensive, Accurate, and Fast Distance-Based Phylogeny Inference Program: Table 1. Mol. Biol. Evol. 2015, 32, 2798-2800. [CrossRef]

30. Farris, J.S. Estimating Phylogenetic Trees from Distancec Matrices. Am. Nat. 1972, 106, 645-668. [CrossRef] 